\title{
DEPRESSION AND CANCER
}

\section{David W. Kissane, Mario Maj y Norman Sartorius (Eds.)}

John Wiley \& Sons, Ltd, Chichester, UK 2011. Doi: 10.1002/9780470972533.ch8

Se expone la comorbilidad entre depresión y cáncer, la necesidad de un adecuado diagnóstico, y los tratamientos más efectivos. Se compone de 9 capítulos (285 páginas), elaborado por 28 reconocidos expertos.

Mary Jane Massie, Mari Lloyd-WiIliams, Greg Irving y Kimberley Miller describen la epidemiología de la depresión en pacientes de cáncer (en el caso de depresión mayor de 3 a 38\%; para síndromes del espectro de la depresión, 1,5 a 52\%). Los tipos de cáncer altamente asociados a depresión incluyen los cerebrales (4193\%), pancreáticos (hasta el 50\%), cabeza y cuello (hasta el $42 \%$ ), mama (4,5-37\%), ginecológicos $(23 \%)$ y pulmonares $(11 \%)$. Asimismo, señalan como la depresión en pacientes con cáncer debe diferenciarse de la desmoralización. La persona deprimida ha perdido la capacidad de sentir placer en general, mientras que la persona desmoralizada puede disfrutar el momento presente si se distrae de los pensamientos desmoralizantes.

David M. Clarke desarrolla un capítulo acerca de la depresión, desmoralización y adaptación psicológica en personas con cáncer La respuesta emocional al cáncer esta determinada por tres factores: a) el modo en que se valora la enfermedad (ej., como un reto o como una amenaza), b) la percepción de control y c) la consideración sobre el pronóstico (bueno o malo). Asimismo, expone las estrategias de adaptación al cáncer (espíritu de lucha, negación, fatalismo, desesperanza y preocupación ansiosa).

Dominique L. Musselman, Andrew $\mathrm{H}$. Miller, Erica B. Royster y Marcia D. McNutt exploran las relaciones de la biología, citoquinas y depresión en cáncer. En concreto describen como las citoquinas proinflamatorias (TNF-alpha, IL-1 y IL-6) inducen conductas de enfermedad semejantes a la depresión mayor. El síndrome incluye anhedonia, deterioro cognitivo, ansiedad/ irritabilidad, enlentecimiento psicomotor, fatiga, anorexia, alteraciones del sueño y sensibilidad aumentada al dolor. Las citoquinas pro-inflamatorias se encuentran elevadas en los pacientes con cáncer y depresión y sus niveles se correlacionan con las conductas de enfermedad.

Steven D. Passik y Amy E. Lowery exponen los métodos de screening y reconocimiento de la depresión en pacientes de cáncer. La prevalencia de la depresión en cáncer es muy variada en cuanto al rango, que refleja de las diferencias entre los estudios (diferentes definiciones de depresión, el uso de cuestionarios de auto-informe o entrevistas psiquiátricas, el tipo de cáncer o el estadio, o los tratamientos del cáncer). El diagnóstico de depresión mayor en pacientes con cáncer presenta un reto, ya que los criterios diagnósticos del DSM-IV incluyen varios síntomas que se superponen a los síntomas propios del cáncer o de los efectos secundarios del tratamiento (pérdida del apetito, disminución de peso, trastornos del sueño, fatiga, falta de ener- 
gía, dificultad para concentrarse, enlentecimiento psicomotor). La depresión es difícil de evaluar en pacientes con cáncer, ya que sus síntomas se presentan en un continuo que va desde los sentimientos normales de tristeza a un trastorno afectivo mayor. La atención oncológica se concentra en los aspectos físicos y los síntomas emocionales pueden ser pasados por alto o incluso no ser tomados en cuenta por considerarse consecuencias esperadas del cáncer. Los pacientes podrían presentarse reacios a acudir a sus médicos por una dolencia emocional, ya sea por miedo a que ello distraiga a éste de sus esfuerzos curativos, o por temor a actitudes culturales negativas hacia la depresión. Se exponen las principales herramientas de screening para depresión en pacientes con cáncer.

M. Robin DiMatteo y Kelly B. HaskardZolnierek describen los mecanismos a través de los cuales la depresión impacta la adherencia a los tratamientos y la supervivencia del cáncer. Los principales son la incapacidad para integrar el diagnóstico de cáncer y la información sobre el tratamiento, la motivación reducida para el autocuidado, la dificultad para la planificación, las creencias negativas con respecto a la salud y el pesimismo sobre el tratamiento, la evitación de conductas que favorecen a la salud, el aislamiento social, el uso reducido de los recursos comunitarios, y la mayor dificultad para tolerar los efectos adversos del tratamiento.

William Breitbart, Hayley Pessin y Elissa Kolva exponen el tema del suicidio. Los pacientes con cáncer presentan un riesgo aproximadamente dos veces mayor de cometer suicidio que la población general. La depresión es un factor conocido en al menos la mitad de todos los suicidios, y los individuos que sufren de depresión po- seen un riesgo 25 veces mayor de suicidio. Factores adicionales para el suicidio en pacientes con cáncer incluyen sentimientos como el de sentirse una carga para otros, la pérdida de autonomía, el deseo de controlar la propia muerte, síntomas físicos, desesperanza, preocupaciones existenciales, la ausencia de apoyo social, y el miedo al futuro. Los autores exponen los protocolos de evaluación e intervención en suicidio.

Luigi Grassi, Maria Giulia Nanni, Yosuke Uchitomi y Michelle exponen los aspectos relativos a la medicación antidepresiva y su uso en pacientes con cáncer. Recomiendan comenzar el tratamiento con dosis bajas seguido de un período de incremento de dosis para alcanzar una óptima respuesta individual (las dosis bajas ayudan a evitar los efectos adversos iniciales, particularmente en pacientes en mala condición física). Informar y tranquilizar al paciente en lo que respecta al período de latencia y los posibles efectos adversos, a fin de evitar abandonos prematuros, especialmente si los pacientes están recibiendo otras medicaciones. Tratar al paciente por 4-6 meses a fin de evitar recaídas o nuevos episodios de depresión luego de la remisión. Registrar regularmente las variables físicas del paciente y el uso concomitante de medicaciones para el cáncer (ej., esteroides, antieméticos, antibióticos, antiestrógenos y agentes quimioterapéuticos). Suspender las medicaciones disminuyendo las dosis en $50 \%$ cada dos semanas para reducir el riesgo de síntomas de discontinuación que pueden ser angustiosos y pueden ser confundidos con síntomas propios del cáncer o con una recaída en la depresión. El reconfortar y educar a los pacientes es extremadamente importante en contextos oncológicos. 
David W. Kissane, Tomer Levin, Sarah Hales, Christopher Lo y Gary Rodin, exponen las intervenciones psicológicas en primer lugar la terapia cognitiva, adaptada al pacientes de cáncer, y a continuación el Managing Cancer and Living Meaningfully (CALM) Therapy para pacientes con cáncer avanzado.

Por ultimo, Christoffer Johansen, Susanne Oksbjerg Dalton y Pernille Envold Bids- trup exponen las influencias culturales en las respuestas emocionales y la depresión ante el cáncer.

En definitiva, es un texto que todos los clínicos e investigadores de la Psicooncología deben estudiar y consultar.

Juan A. Cruzado

Universidad Complutense de Madrid 